\section{A good addition}

TePe has expanded its sustainably produced $\mathrm{TePe}$ GOOD range with the introduction of the TePe GOOD Compact toothbrush and TePe GOOD Tongue Cleaner the UK's first bio-plastic tongue cleaner.

The TePe GOOD Compact toothbrush and TePe GOOD Tongue Cleaner are produced using renewable raw materials, sugar cane and castor oil. This enables TePe to recirculate up to $95 \%$ of the $\mathrm{CO}_{2}$ emissions during the products' life cycle, offering a more sustainable choice to consumers and the industry, without compromising on product quality, design or hygiene.

As with all TePe products, the TePe GOOD Compact toothbrush and TePe GOOD Tongue Cleaner are manufactured using $100 \%$ green energy at TePe's Swedish Malmö factory, which has the largest solar power plant in the city.

In addition to its sustainability benefits, the TePe GOOD Compact toothbrush has been developed in collaboration with dental experts to ensure a high-quality design, featuring an ergonomic handle, tapered head and rounded filaments for maximum cleanliness. The TePe GOOD Compact toothbrush is recommended for children and adults who prefer a smaller brush head.

Furthermore, the new TePe GOOD Tongue Cleaner has been carefully designed to remove bacteria from the tongue, helping to prevent bad breath and improve oral health. It corresponds to the natural shape of the tongue, making it easier to reach far back in the mouth, while the slim shape reduces the gag reflex. What's more, with three slightly raised cleaning surfaces for a triple-effect, one stroke should be enough to clean the tongue efficiently.

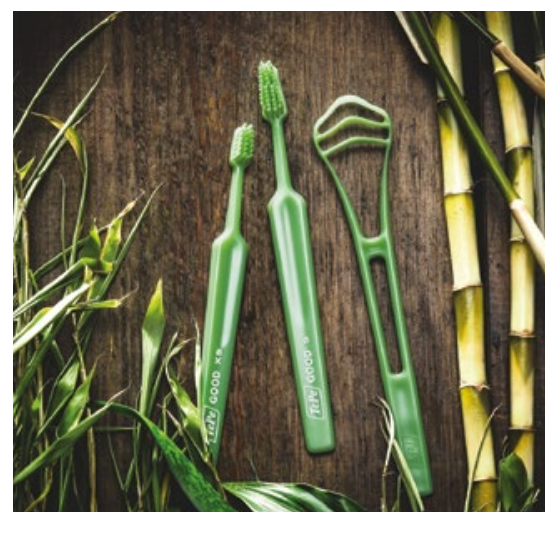

\section{Bring their smile back to life!}

Fully edentulous patients are likely to suffer from a lack of confidence.

Give them a reason to smile again by recommending the All-on- 4 treatment concept from Nobel Biocare.

An innovative solution for edentulous and soon to be edentulous patients, the All-on-4 treatment concept has revolutionised how professionals provide full arch restoration. Utilising just four tilted implants, not only does the concept not require any bone grafting, but it can also provide patients with a full fixed prosthesis on the day of surgery. This helps to restore patients' confidence sooner.

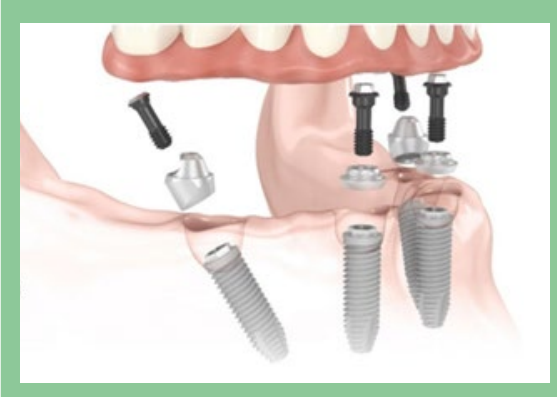

Alongside this, the All-on- 4 treatment concept is one of the more cost-effective treatments for the fully edentulous jaw.

It's little wonder that $98 \%$ of patients would recommend the treatment to someone they know...

Find out more about the All-on- 4 treatment concept by contacting Nobel Biocare on 0208 7563300 , or visit www.nobelbiocare.com.

\section{A real diamond}

Dress like a star with the Grey's Anatomy

Collection of scrubs.

Distributed by Diamond Designs, the collection is a high-quality, stunning choice of uniform for any dental team who believes that looking great can help you to perform even better.

Beautifully constructed in four-way stretch, feather light fabric, the Grey's Anatomy Collection is perfect for practitioners on the go. All the pieces balance style and comfort and will give your team a professional, modern image.

This range is just one of the uniform collections available from Diamond Designs. Contact the team today to discover your ideal fit.

To find out more, visit www.

diamonddesignsuniforms.com.

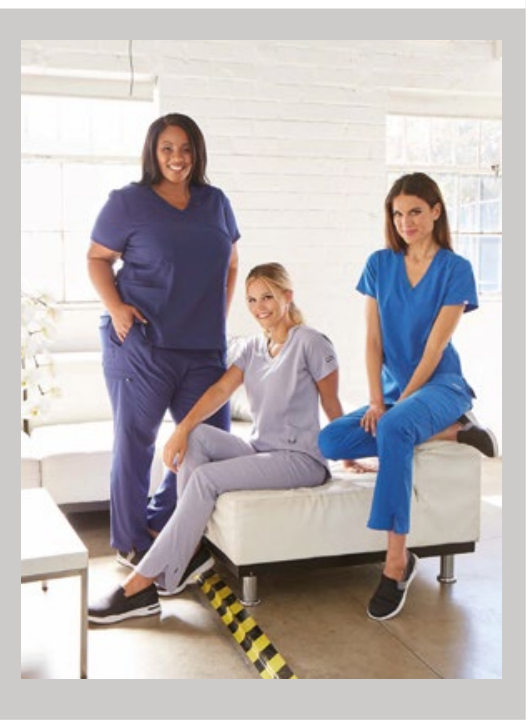

\section{A great grip}

Oral hygiene aids which are comfortable to hold and easy to manipulate within the oral cavity, can help patients optimise their daily routine.

TANDEX produces ergonomically friendly oral-hygiene aids that they will find comfortable to use.

TANDEX brushes have a long-angled neck, with a handle that ensures a good grip. The TANDEX FLEXI interdental brush has also been created for optimal functionality, being flexible and stable, to efficiently reach spaces around the back molars. The SOLO interspace brush also ensures access to critical points, thanks to its clever design.

For more information on Tandex's range of products visit www.tandex.dk.
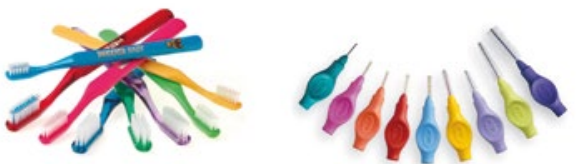